\title{
Evaluation du risque infectieux en chirurgie orale chez les patients sous anti-tnf alpha
}

\section{Pannérec V, Catros S, Fricain JC. (UFR d'Odontologie, Bordeaux)}

Le TNF alpha (Tumor Necrosis Factor) est une cytokine pro-inflammatoire, médiateur de l'immunité initialement utilisée comme thérapie anti-cancéreuse (Moiton.2002).

Cette molécule est essentiellement sécrétée par les cellules du système réticuloendothélial, sous la forme d'un précurseur le pro-TNF qui, grâce à l'action d'une métalloprotéase, forme le TNF alpha.

Les anti-TNF alpha sont des traitements récents (Première AMM en 1999) appartenant à la classe des biothérapies. Ces molécules ont initialement montré leur efficacité dans la Polyarthrite Rhumatoïde et la maladie de Crohn avec une amélioration clinique, pronostique et de la qualité de vie des patients. Les indications se sont élargies aux Maladies Inflammatoires Chroniques de l'Intestin, au Psoriasis, à la Granulomatose de Wegener (Grosdemanche 2011). Une enquête menée auprès de 96 médecins d'Eure et Loire a montré que 87.5\% d'entre eux avaient au moins un patient concerné (Grosdemange 2011).

D'utilisation récente et de plus en plus fréquente, les anti-tnf alpha sont mal connus des praticiens et la conduite à tenir a récemment été codifiée (recommandations SFCO). L'objectif de ce travail est donc de réaliser une mise au point sur le risque infectieux en chirurgie orale chez des patients traités par ces médicaments. Dans un premier temps nous présenterons les anti-TNF alpha et leur mode d'administration intraveineux ou sous-cutané. Ensuite nous préciserons les connaissances actuelles quant aux risques infectieux per et post opératoires en chirurgie générale et orale.

Des complications ont été rapportées chez des patients traités par anti-TNF alpha (études pivot de mise sur le marché), en raison de la modification de la réponse inflammatoire induite par ces traitements. Différents types d'infections communautaires ou opportunistes, bactériennes ou virales, fongiques ou parasitaires ont été décrites dans la littérature (Andre.2010). Les données de l'observatoire national prospectif pluridisciplinaire RATIO de 2003 à 2007 ont répertorié les infections opportunistes et concluent à une proportion exagérée de germes à multiplication intracellulaire, à un risque relatif moyen de 2 et qu'à peine $2 \%$ des patients font une infection grave. Quant aux données du registre CORRONA (Consortium of Rheumatology Researchers of North America) elles permettent de conclure à l'existence d'un risque infectieux légèrement plus élevé pour les patients sous anti TNF alpha par rapport à un groupe contrôle (RR :1,16, IC95\%, 1,06; 1,$28 ; p=0,002)$.

En Août 2012 la Société Française de Chirurgie Orale a publié des recommandations sur la stratégie à adopter afin d'éviter les infections post-opératoires chez les patients sous anti-TNF alpha. II est recommandé d'arrêter les antiTNF alpha dans des délais suffisants en cas d'intervention chirurgicale (hors implantologie) pour limiter le risque infectieux (Etanercept au moins 2 semaines, Infliximab et Adalimumab au moins 4 semaines) et de contacter le médecin prescripteur.

Cette revue systématique a révélé que le risque infectieux semble difficile à établir mais moins élevé dans « la vraie vie » que dans les études randomisées contrôlées. Aussi, des mesures simples de prévention et d'évaluation du risque infectieux relatif à la pathologie systémique initiale sont proposées en complément des recommandations qui, en l'absence de preuve restent à suivre.

PANNEREC Virginie pnnrcvrgn83@aol.fr

This is an Open Access article distributed under the terms of the Creative Commons Attribution License 2.0, which permits unrestricted use, distribution, and reproduction in any medium, provided the original work is properly cited. 